semi-insulating InP substrates with $\mathrm{SC}$ mirrors having threshold currents and quantum efficiencies comparable to those with conventionally cleaved mirrors. Finally, since the $\mathrm{SC}$ technique uses the conventional cleaving procedures, it is well suited for mass production.

We would like to thank A. Savage for his help in the contact metallization procedure.

'I. Ury, K. Y. Lau, N. Bar-Chaim, and A. Yariv, Appl. Phys. Lett. 41, 126 (1982).

${ }^{2}$ N. Bar-Chaim, K. Y. Lau, I. Ury, and A. Yariv, paper TuD 1-1 presented at Topical Meeting on Integrated and Guided-Wave Optics, Florida 1984.
${ }^{3}$ H. Matsueda, S. Sasaki, and M. Nakamura, IEEE J. Lightwave Tech. LT1, $261(1983)$.

${ }^{4}$ K. Kishino, Y. Suematsu, K. Utaka, and H. Kawanishi, Jpn. J. Appl. Phys. 17, 589 (1978).

'J. L. Merz, R. A. Logan, and A. M. Sergent, IEEE J. Quantum Electron. QE-15, 72(1979).

${ }^{6}$ L. A. Coldren, K. Iga, B. I. Miller, and J. A. Rentschler, Appl. Phys. Lett. 37, $681(1980)$.

${ }^{7}$ H. Blauvelt, N. Bar-Chaim, D. Fekete, S. Margalit, and A. Yariv, Appl. Phys. Lett. 40, 289 (1982).

${ }^{8}$ O. Wada, S. Yamakoshi, T. Fuji, S. Hiyamizu, and T. Sakurai, Electron. Lett. 18, $189(1982)$.

${ }^{9}$ B. F. Levine, J. P. van der Ziel, R. A. Logan, and C. G. Bethea, Electron. Lett. 18, $690(1982)$.

${ }^{10}$ B. F. Levine (private communication).

\title{
Coherence and focusing properties of unstable resonator semiconductor lasers
}

\author{
M. Mittelstein, J. Salzman, T. Venkatesan, a) R. Lang, and A. Yariv \\ California Institute of Technology, Pasadena, California 91125
}

(Received 26 November 1984; accepted for publication 25 February 1985)

The emission characteristics of unstable resonator semiconductor lasers were measured. The output of an $80-\mu \mathrm{m}$-wide laser consists of a diverging beam with a virtual source $5 \mu \mathrm{m}$ wide located $50 \mu \mathrm{m}$ behind the laser facet. A high degree of spatial coherence of the laser output was measured, indicating single lateral mode operation for currents $I \leqslant 3 I_{\text {th }}$.

In semiconductor lasers, the resonant cavity is generally formed by two flat, parallel (cleaved) mirrors (a FabryPerot cavity). Such a resonator lies on the boundary between the stable and unstable regions of the stability diagram of optical resonators. ${ }^{1}$ However, the high single-pass-gain characteristic of semiconductor lasers suggests that an unstable resonator (UR) cavity may be advantageous, since this cavity provides a large mode volume and a substantial discrimination against higher order lateral modes. ${ }^{2}$ Furthermore, the self-focusing behavior of lasing filaments can be counterbalanced by the magnifying effect of the cavity, making it possible to obtain higher optical output powers.

Preliminary experiments with semiconductor lasers, in which one mirror was cleaved and the other mirror was convex, have been reported. ${ }^{3,4}$ Recently, we demonstrated the operation of GaAlAs-GaAs lasers with two convex mirror surfaces with no planar feedback in any part of the cavity. ${ }^{5}$ By measuring the near field distribution of these lasers, we showed that the filamentation problems were overcome. Furthermore, high output power $(0.35 \mathrm{~W})$ in a stable highly coherent lateral mode and relatively high external quantum efficiency $\eta_{d}=0.22$ were demonstrated in these devices. ${ }^{5}$

In this letter, we report on additional measurements aimed at showing that the UR semiconductor laser radiation consists of a diverging, single lateral mode that can, in prin-

\footnotetext{
"Visiting Associate at Cal Tech from Bell Communications Research,
} Murray Hill, New Jersey 07974. ciple, be focused into a spot of $\leqslant 5 \mu \mathrm{m}$ in diameter. The UR laser is shown (drawn to scale) in Fig. 1(b). The symmetric unstable resonator is defined by etched facets with a radius of curvature of $R=240 \mu \mathrm{m}$ and at a length of $L=250 \mu \mathrm{m}$ on a double heterostructure GaAs-GaAlAs wafer. The gain region is defined by a metal contact whose width is $W=80 \mu \mathrm{m} .^{5}$

Since the output beam of the UR laser is expected to be highly astigmatic, we investigated the minimum spatial extension of the beam in the principal planes. In the plane perpendicular to the $p-n$ junction, the beam waist is at the facet, whereas in the plane of the junction, the light appears to originate at a virtual source located deep behind the laser facet. This implies that the waveguiding effects determine the beam characteristics in the direction perpendicular to the $p-n$ junction, whereas the cylindrical UR governs the beam characteristics in the plane of the junction.

The depth of this virtual source ( $S^{\prime}$ in Fig. 1) was measured with the aid of a microscope objective, and a value of $S^{\prime}=50 \pm 5 \mu \mathrm{m}$ was obtained. The intensity distribution of this virtual source in the plane of the $p-n$ junction was studied as a function of injection current, and a dramatic narrowing was observed when the injection current was increased from threshold $\left(I_{\mathrm{th}}\right)$ at $I \simeq 700 \mathrm{~mA}$ to $3 I_{\mathrm{th}}$ (Fig. 2). At $3 I_{\mathrm{th}}$, the measured width of this virtual source (FWHM) was $5 \mu \mathrm{m}$ with negligible intensity in the wings.

These results can be explained with the aid of the fol- 

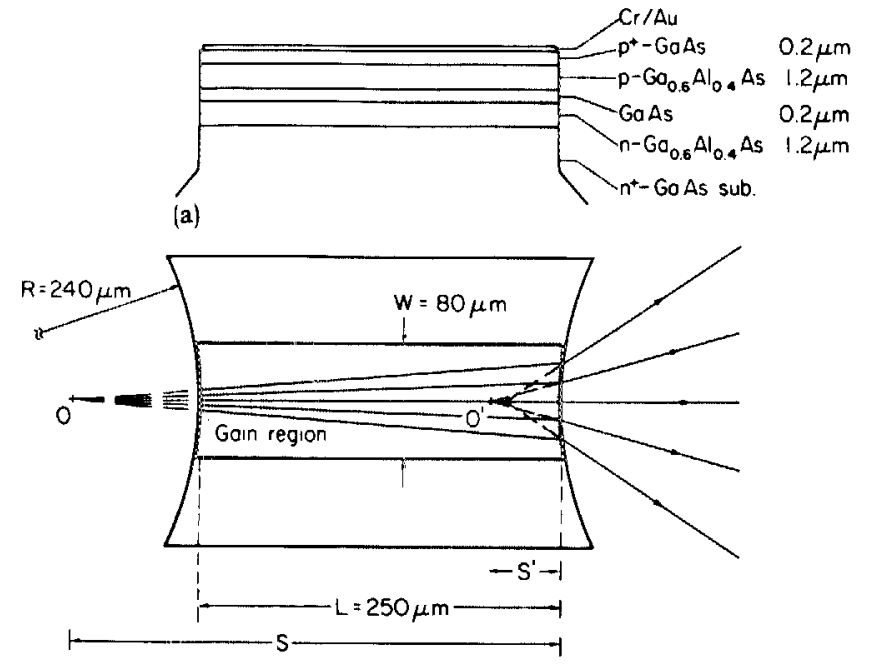

(b)

FIG. 1. (a) Schematic of the layer structure. (b) Top view of the laser with geometrical-ray description of the UR semiconductor laser. Radiation emanating from a virtual source at point $O$ is refracted through the output mirror, resulting in a virtual source of the output beam at point $O^{\prime}$. Shown also is the spherical aberration at $O^{\prime}$.

lowing simple model: The light inside the UR that propagates toward the output-coupling mirror (Fig. 1) can be described in the geometrical optics approximation as a spherical wave originating at a point $O$, whose distance from the exit facet ${ }^{2}$ is

$$
S=\llbracket R L / 2+(L / 2)^{2} \rrbracket^{1 / 2}+L / 2=339 \mu \mathrm{m},
$$

where $R$ is the magnitude of the radius. After refraction at the cylindrical surface, we obtain for the depth of the virtual source point in the small angle approximation, ${ }^{6}$

$$
S^{\prime}=[(n-1) / R+n / S]^{-1}=48 \mu \mathrm{m},
$$

where the index or refraction of the laser medium is $n=3.5$. This result is in very good agreement with the measured value. The apparent sharpening of the intensity distribution with increasing injection current at the virtual source plane is expected from the fact that below threshold, the light output is due mainly to spontaneous emission and amplified

(a)

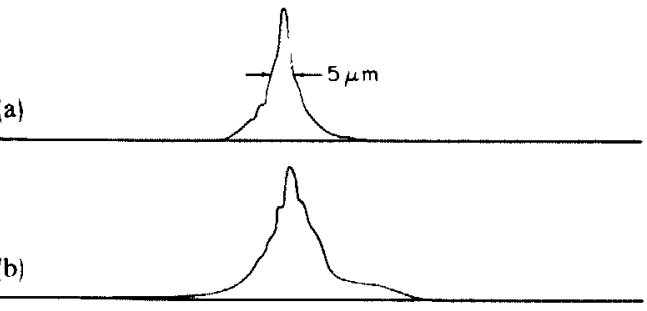

(c)
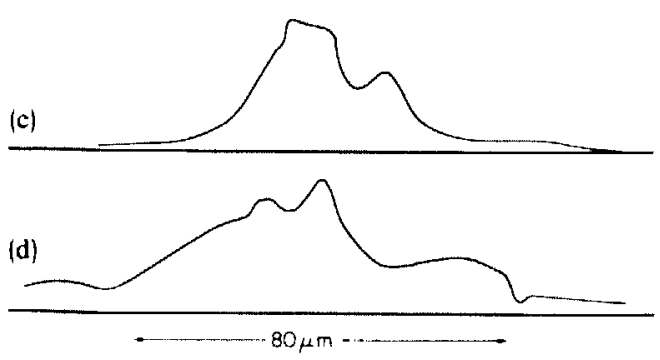

FIG. 2. Normalized intensity profiles at the virtual source plane as a function of injection current $I$. (a) $I=3 I_{\mathrm{th}} ;$ (b) $I=1.9 I_{\mathrm{ih}} ;(\mathrm{c}) I=1.4 I_{\mathrm{th}} ;$ (d) $I \simeq I_{\text {th }}$.
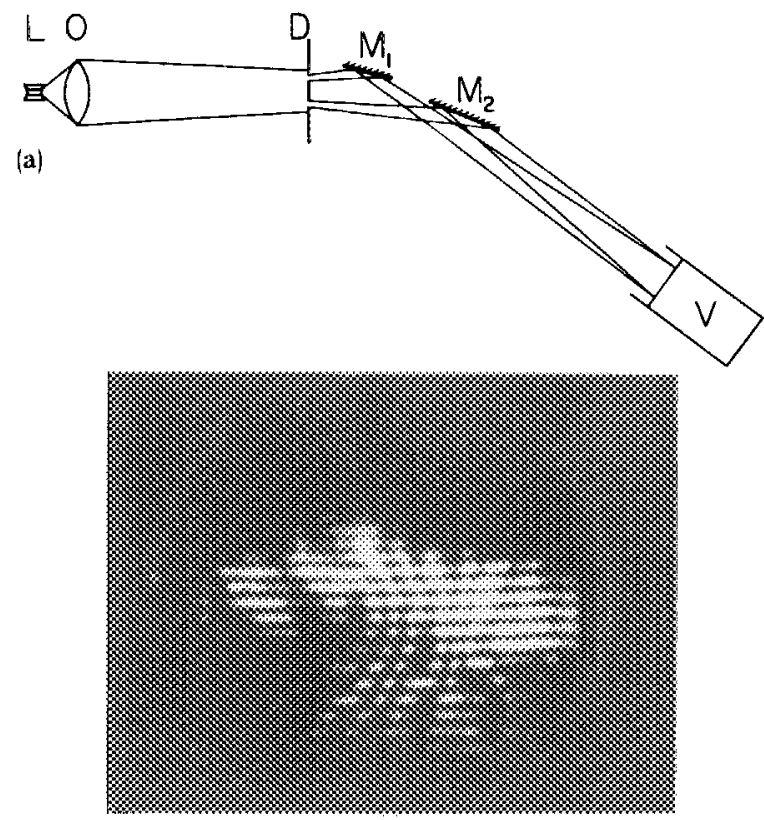

(b)

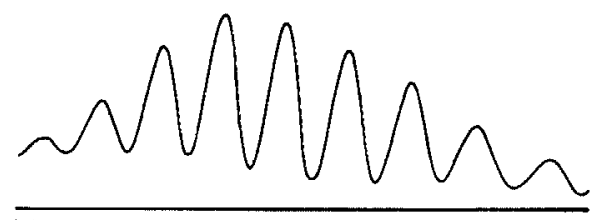

(c)

FIG. 3. Double slit interference experiment. (a) Schematic of the optical layout; $L=U R$ laser; $O=$ microscope objection; $D=$ double slit; $M_{1}, M_{2}=$ plane mirrors; $V=$ vidicon camera. (b) Photograph of interference pattern. $(c)$ Intensity trace of interference pattern.

spontaneous emission, which is not influenced by the resonator feedback. At higher currents, the feedback effect dominates and the laser output exhibits the mode properties of the unstable resonator. It should be noted that the FWHM of the virtual source in the lasers reported here is affected by a strong spherical aberration. A sharper image may be obtained from an aspherical output coupler.

The lateral mode characteristics of the UR laser can be studied by measuring the spatial degree of coherence of the output beam as a function of lateral position. In the multimode case, high spatial coherence is expected only between two points corresponding to comparable intensities of the same lateral mode, whereas two points whose intensities correspond to different modes will exhibit a low degree of spatial coherence. Spatial coherence between two points can be measured by observing the fringe visibility function (FVF) in an interference pattern produced by the radiation transmitted through two narrow shits at these points (Young's double slit experiment). By scanning the slits across the near field of the laser, the coherence function between any two points on the laser output facet can be obtained.

In our experiment (Fig. 3), the laser near field was imaged and magnified to the plane $D$, which contained the double slit screen. The slit separations and their positions relative to the laser near field could be varied. The beam exiting from each slit was nearly diffraction limited. Due to the angular separation between these two beams, additional mirrors were required in order to produce two overlapping 
(and interfering) beams, as shown in Fig. 3(a). A high degree of spatial coherence was observed over the entire spatial extent of the laser output, without dependence on lateral position. Figures $3(b)$ and $3(c)$ are examples of the interference patterns recorded with a FVF of $\sim 0.6$. When the injection current was increased over $3 I_{\text {th }}$, the fringe visibility function decreased, presumably indicating the onset of higher order modes. Similar measurements with broad area lasers with cleaved facets (Fabry-Perot cavity), fabricated from the same wafer, showed substantially lower visibility of the interference pattern and a spatial degree of coherence varying with position and injection current. This provides strong evidence that the UR structure results in a single lateral mode at high injection currents in spite of the very wide $(80 \mu \mathrm{m})$ width of the laser mode.

In conclusion, we have measured some of the emission characteristics of UR lasers. ${ }^{5}$ The output beam is highly astigmatic, with a virtual source corresponding to that expected from the modal analysis of unstable resonators. Single mode operation was demonstrated by measuring the spatial degree of coherence of the laser output. If the highly astigmatic beam is corrected by appropriate external optics, the focusing capability of these lasers may lead to power densities in a single mode higher than that emitted by any other semiconductor sources reported until now.

This research is supported by grants from the Air Force Office of Scientific Research and the Office of Naval Research. J.S., R.L. and M.M. would like to acknowledge the support of the Bantrell Postdoctoral Fellowship, the Nationat Science Foundation, and the German National Scholarship Foundation, respectively.

'G. D. Boyd and H. Kogelnik, Bell Syst. Tech. J. 41, 1347 (1962).

${ }^{2}$ A. E. Siegman, Proc. IEEE 53, 277 (1965).

${ }^{3}$ A. P. Bogatov, P. G. Eliseev, M. A. Man'ko, G. T. Mikaelyan, and Yu N. Popov, Sov. J. Quantum Electron. 10, 620 (1980).

${ }^{4}$ R. R. Craig, L. W. Casperson, G. A. Evans, and J. J. J. Yang, presented at Conference on Lasers and Electro-optics, Anaheim, CA, June 1984.

${ }^{5} \mathrm{~J}$. Salzman, T. Venkatesan, R. Lang, M. Mittelstein, and A. Yariv, Appl. Phys. Lett. 46, 218 (1985).

'M. Born and E. Wolf, Principles of Optics (Pergamon, London, 1965), Chap. 4.4, pp. $157-160$.

\title{
Power enhancement for argon narow tube lasers by a transverse magnetic 解eld
}

\author{
Shigeaki Kobayashi and Takeshi Kamiya \\ Department of Electronic Engineering. University of Tokyo, 7-3-1. Hongo, Bunkyo-ku, Tokyo 113, Japan \\ Toshiharu Hayashi and Tatsumi Goto \\ Manufacturing Engineering Laboratory, Toshiba Corporation, 8, Shinsugita-cho, Isogo-ku, Yokohama 235, \\ Japan
}

(Received 17 January 1985; accepted for publication 6 March 1985)

\begin{abstract}
Appreciable enhancement in output and power efficiency for compact Ar II lasers $(488.0 \mathrm{~nm})$ is obtained by applying a transverse magnetic flux density at approximately $700 \mathrm{G}$. The enhancement factor amounts to as large as 3 in laser output and the efficiency becomes more than twice as much as without magnetic flux. A sharp rise in output occurs above the critical magnetic flux density, $450 \mathrm{G}$ in the present work, where the cyclotron radius of electrons is nearly equal to one-half of the tube radius.
\end{abstract}

There is a wide range of industrial as well as scientific demand for blue and green coherent light sources with small drive power levels, such as in laser printing, peripherals of data processing machines, chemical and biological material analysis. At present, compact Ar II lasers are almost the only choice which satisfies the requirements for $\mathrm{cw}$ output on approximately several tens of milliwatt levels with electrical power supplies smaller than $1 \mathrm{~kW}$. If it is desired to achieve a still more compact device which operates at a milliwatt output power level with a power supply well below the kilowatt level, the major difficulty is the decreasing conversion efficiency for decreasing the operating power levels, compared with Watt-class Ar II lasers.

This letter presents an appreciable enhancement in output power for compact Ar lasers $(488.0 \mathrm{~nm})$ achieved by applying approximately $700 \mathrm{G}$ of transverse magnetic flux density. The enhancement factor becomes as large as 3 . The characteristics and physical mechanism for the observed effect are found to be completely different from the longitudinal magnetic field effect. ${ }^{\prime}$

The laser tubes under investigation are a slight modification of the compact argon ion lasers, Toshiba LAI-106, which have a capillary section $1 \mathrm{~mm}$ in diameter, $36 \mathrm{~mm}$ in length, and 1.0 Torr gas filling pressure.

Figure 1(a) shows the light output versus magnetic flux density plot with the discharge current level as the parameter for a linearly polarized laser with external mirrors. A sharp output power rise occurs at above $450 \mathrm{G}$, which is hereafter called the critical magnetic flux density. In Fig. $1(b)$, the tube voltage is plotted as a function of magnetic flux 